\title{
Persistence of specific bactericidal antibodies at 5 years of age after vaccination against serogroup $B$ meningococcus in infancy and at $\mathbf{4 0}$ months
}

Fiona McQuaid MBBS, Matthew D. Snape MBBS MD, Tessa M. John BSc, Sarah Kelly MSc, Hannah Robinson RN, Ly-Mee Yu MSc, Daniela Toneatto MD, Diego D’Agostino MSc, Peter M. Dull MD, Andrew J. Pollard PhD

\begin{abstract}
- ABSTRACT
Background: The multicomponent serogroup B meningococcal (4CMen $B$ ) vaccine induces antibodies against indicator strains of serogroup $B$ meningococcus under various schedules. We investigated the persistence of antibodies in 5-year-old children 18-20 months after their last dose (at about 3.5 years of age).

Methods: We assessed 5-year-old children who received the $4 C M e n B$ vaccine or a recombinant protein vaccine in a previous randomized trial. We also recruited 50 vaccine-naive 5 -year-olds and administered 2 doses of 4 CMenB to each child. We measured serum bactericidal antibody titres against 4 indicator strains of serogroup $B$ meningococcus matched to each individual vaccine component and against 4 mismatched strains.
\end{abstract}

Results: Of those who received the $4 C M e n B$ vaccine at $2,4,6,12$ and 40 months $(n=16)$, the percentage with protective antibody titres $(\geq 1: 4)$ at 60 months ranged from $44 \%$ to $88 \%$ against matched strains and from $13 \%$ to $81 \%$ against mismatched strains. Loss of protective titres was also observed for those who received the $4 C M e n B$ vaccine at 12,40 and 42 months $(n=5)(80 \%-100 \%$ against matched strains, $60 \%-100 \%$ against mismatched strains) or at 40 and 42 months $(n=29)(31 \%-100 \%$ against matched strains, $41 \%-81 \%$ against mismatched strains). Administering the $4 C M e n B$ vaccine to 5-year-old children yielded protective titres against matched strains in $92 \%$ $100 \%$ and against mismatched strains in $59 \%$ $100 \%$. The majority of these children reported injection-site pain (40/50 [80\%] after dose 1, $39 / 46$ [85\%] after dose 2) and erythema (47/50 [94\%] and 40/46 [87\%], respectively); rates of fever were low (5/50 [10\%] and 2/46 [4\%], respectively).

Interpretation: Waning of immunity by 5 years of age occurred after receipt of the 4CMenB vaccine in infancy, even with an additional booster at 40 months. The $4 \mathrm{CMenB}$ vaccine is immunogenic and was fairly well tolerated by 5-year-old children, although injection-site pain was noteworthy. Trial registration: Clinical Trials.gov, no. NCT01027351
$\mathrm{T}$ he multicomponent serogroup B meningococcal (4CMenB) vaccine is licensed in the European Union, Australia and Canada to prevent serogroup B meningococcal disease. It was developed using "reverse vaccinology," in which candidate antigens were identified by interrogating the whole meningococcal genome. ${ }^{1}$ The $4 \mathrm{CMenB}$ vaccine consists of 3 surface proteins (factor $\mathrm{H}$ binding protein [fHbp], Neisseria adhesin A [NadA] and Neisseria heparin-binding antigen [NHBA]), along with a fourth component, the outer membrane vesicle, which acts as both antigen and adjuvant. ${ }^{1}$

Group B meningococcal disease is a potentially devastating condition, with an average case fatality rate of $5.2 \%$ (data for England and $\mathrm{Wales}^{2}$ ), and over a third of survivors are left with measurable functional deficits. ${ }^{3}$ The incidence of laboratory-confirmed cases is about 1 per 100000 population in England ${ }^{4}$ and 0.33 per 100000 population in Canada. ${ }^{5}$ The recommendation of the United Kingdom Joint Committee on Vaccination and Immunisation that the $4 \mathrm{CMenB}$ vaccine be introduced into the routine UK immunization schedule should, if implemented, lead to a reduction in this morbidity and mortality. ${ }^{6}$ Data on the persistence of antibody responses following infant or toddler immunization, and after subsequent boosting, remain limited yet will be important for guiding implementation of this recommendation.
Competing interests: See end of article for competing interests.

This article has been peer reviewed.

Accepted: Mar. 6, 2015 Published: Mar. 23, 2015

Correspondence to: Fiona McQuaid, fiona.mcquaid@ed.ac.uk

CMAJ 2015. DOI:10.1503 /cmaj.141200 
We present here the results of a follow-on study investigating the persistence of antibodies 18-20 months after the last dose in 5-year-old children previously immunized under a variety of schedules with $4 \mathrm{CMenB}$ vaccine or another investigational vaccine (recombinant protein serogroup B meningococcal [rMenB] vaccine), which lacks the outer membrane vesicle component of the $4 C M e n B$ vaccine. Since the original infant study, ${ }^{7}$ CMenB vaccine has emerged as the preferred vaccine, because addition of the outer membrane vesicle component improves the breadth of strain coverage; ${ }^{8}$ however, the extension study continued follow-up for all of the original children, and all results are therefore presented here.

\section{Methods}

This phase 2, open-label, single-centre extension study ran from January 2010 to August 2012 and was approved by the Oxfordshire Research Ethics Committee B (reference 09/H0605/89).

The primary immunogenicity objective of the extension study was to assess persistence of antibodies at 40 months of age, as reported previously. ${ }^{9}$ Here, we present the secondary outcomes of antibody persistence at 60 months of age and the immunogenicity, safety and tolerability of a 2-dose catch-up regimen of $4 \mathrm{CMenB}$ vaccine administered at 60 and 62 months.

\section{Participants}

In the original infant study, ${ }^{7} 147$ infants from the UK were recruited and randomly assigned, on a 2:2:1:1 ratio, to receive $4 \mathrm{CMenB}$ or rMenB vaccine at 2, 4, 6 and 12 months or to receive one of these vaccines at 12 months alone.

Of these 147 infants, 70 participated in the 40-month extension study, ${ }^{9}$ in which those who originally received 4 doses received 1 additional dose (at $40 \mathrm{mo}$ ) and those who originally received 1 dose received 2 additional doses (at 40 and $42 \mathrm{mo}$ ) of the vaccine originally received. These groups are referred to here as follows: 4CMenB 2,4,6,12,40; rMenB 2,4,6,12,40; 4 CMenB $12,40,42$; and rMenB 12,40,42. A fifth group (referred to as $4 \mathrm{CMenB} 40,42$ ) received 2 doses of $4 \mathrm{CMenB}$ vaccine only at 40 and 42 months. All of these children were invited to participate again at about 60 months of age, and an additional 50 healthy, vaccine-naive controls (referred to as 4 CMenB 60,62) were recruited by mail. The study therefore included a total of 6 groups (see Figure 1).

Exclusion criteria were as follows: any previous meningococcal vaccination or history of meningococcal disease, severe allergy to any component of the vaccine, and any serious chronic or progressive disease or suspected immune system impairment. Administration of other vaccines was not permitted within 30 days before or after meningococcal vaccination, with the exception of the influenza vaccine, which could be given up to 14 days beforehand. Participants were temporarily excluded if they had been febrile over the previous day or had been taking antibiotics in the past 7-14 days.

\section{Study procedures}

For children from the 5 groups who had previously received either the $4 \mathrm{CMenB}$ or $\mathrm{rMenB}$ vaccine, a single 5-mL sample of blood was drawn at about 60 months of age. Children in the sixth, vaccine-naive control group received 2 doses of $4 \mathrm{CMenB}$ vaccine, at 60 and 62 months of age, with 5-mL samples of blood being drawn before the first dose and 1 month after the second dose.

\section{Vaccines}

All vaccines were supplied by Novartis Vaccines (Siena, Italy). The 4CMenB vaccine contained $50 \mu \mathrm{g}$ of NadA protein 3, $50 \mu \mathrm{g}$ of the fusion protein NHBA-genome-derived Neisseria antigen (GNA) 1030 (containing NHBA peptide 2), $50 \mu \mathrm{g}$ of GNA2091-fHbp fusion protein (containing fHbp variant 1.1), $25 \mu \mathrm{g}$ of detoxified outer membrane vesicle from Neisseria meningitidis strain NZ98/254 (expressing Porin A serosubtype P1.4) and $1.5 \mathrm{mg}$ aluminum hydroxide. Details of the rMenB vaccine received by some groups in the earlier studies can be found elsewhere. ${ }^{9}$ All vaccines were $0.5 \mathrm{~mL}$ in volume and were administered into the deltoid muscle of the nondominant arm.

\section{Immunogenicity}

We tested for serum bactericidal antibodies, using human serum as the source of exogenous complement (hSBA), against the following 4 indicator strains: 44/76-SL, 5/99, NZ98/254 and M10713. These strains were chosen because each is mismatched for all but one of the vaccine antigens; therefore, each strain assesses the response to a single vaccine component (strain 44/76-SL for fHbp, strain 5/99 for NadA, strain NZ98/254 for the outer membrane vesicle and strain M10713 for NHBA). ${ }^{10}$ Four additional strains (UKP1.7-2,4, GB101, GB355 and GB364), which reflected UK epidemiology and which had been used in the previous studies, were used to assess the vaccines' ability to induce a cross-reactive hSBA response to genetically diverse meningococcal serogroup B strains. Of note, earlier published studies referred to these strains as M00-242922 (UKP1.7-2,4), 
M01-240101 (GB101), M01-240355 (GB355) and M01-240364 (GB364).

An hSBA titre of 1:4 or greater was used as the correlate of protection, as a postvaccination increase in antibody titre of this magnitude has been shown to correlate with clinical efficacy in previous vaccine trials involving other outer membrane vesicle vaccines. ${ }^{11}$

Assays for hSBA against strains 44/76-SL, 5/99 and NZ98/254 were performed at the laboratory of the Health Protection Agency (Manchester, UK) and those for hSBA against strains M10713, UKP1.7-2,4, GB101, GB355 and
GB364 at the Novartis Vaccine Clinical Serology Laboratory (Marburg, Germany).

\section{Reactogenicity and safety data}

Reactogenicity data for the 4CMenB 60,62 group were collected for 7 days after each vaccination using paper diary cards that were completed by parents. These cards listed potential local and systemic reactions, based on previous vaccine studies ${ }^{7,9}$ and graded from mild to severe according to interference with normal activities. Additional space was provided for parents to record any other symptoms. Adverse events

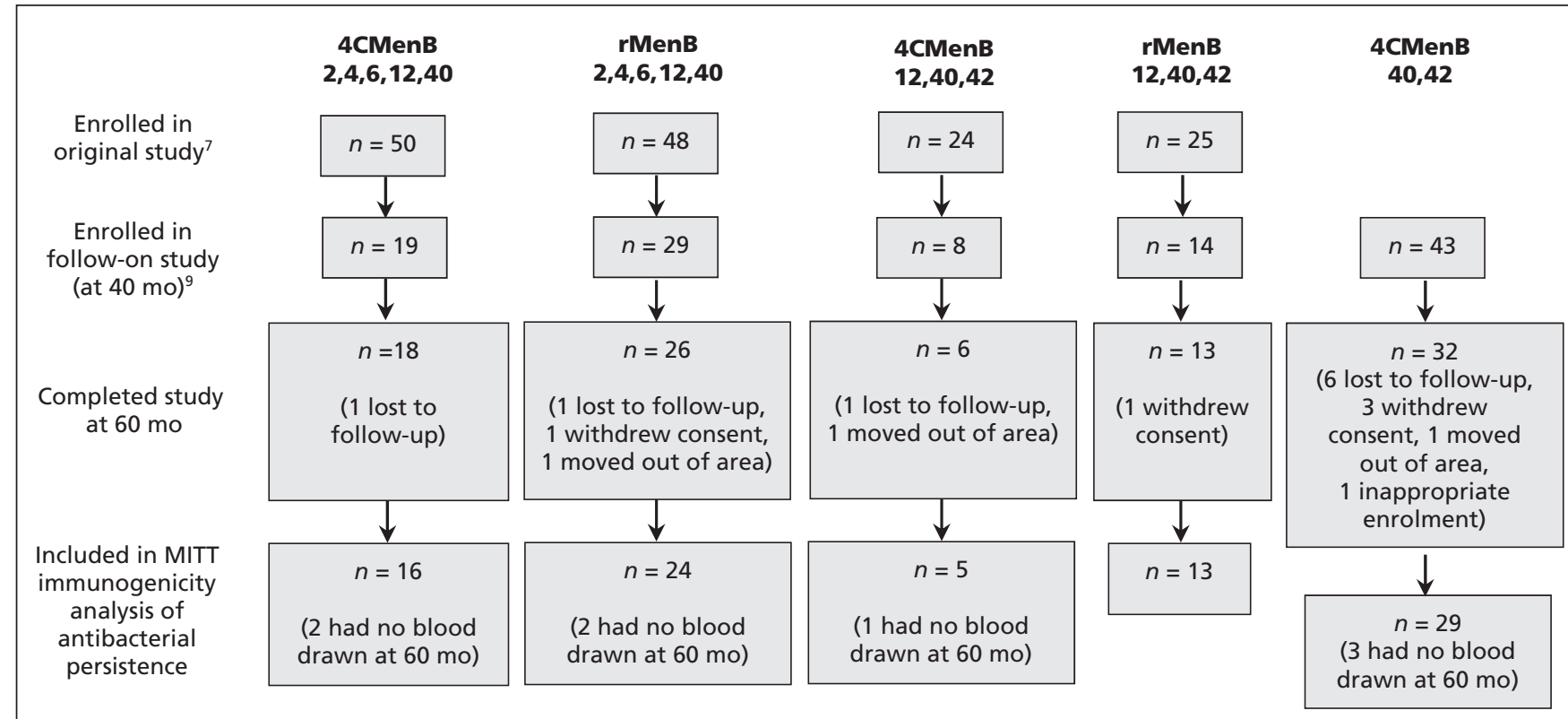

4CMenB 60,62

(vaccine-naive at entry)

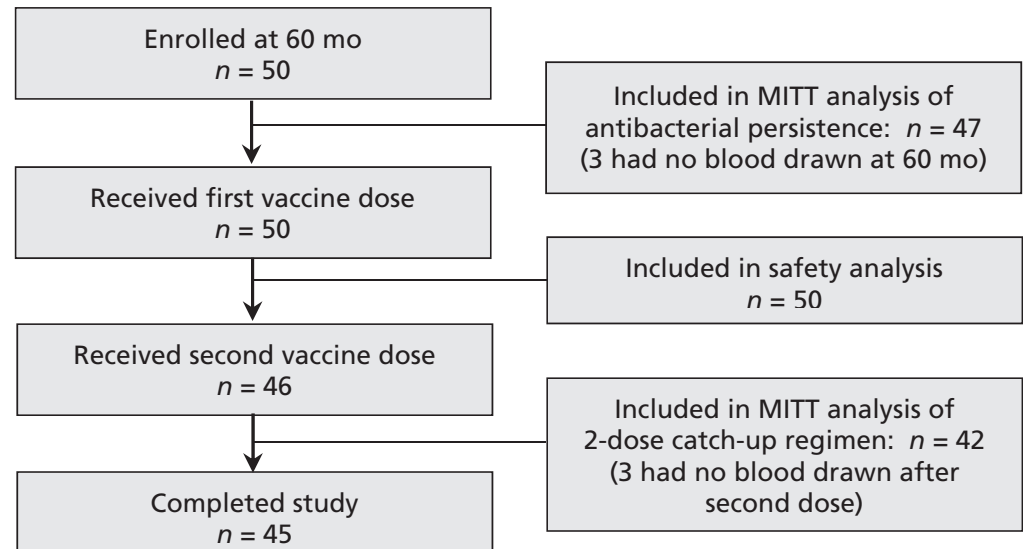

Figure 1: Number of participants enrolled at each time point. In the "4CMenB 60,62 " group, a total of 5 participants withdrew after starting the study: 1 participant withdrew consent because of both vaccine-related pain and distress related to the blood test; 3 participants withdrew because of distress at the second vaccine visit, and the second dose was therefore not given; and 1 participant did not wish to continue after receiving the second vaccine and withdrew at that time. $4 C$ MenB $=$ multicomponent serogroup $B$ meningococcal vaccine, MITT = modified intention-to-treat, rMenB = recombinant protein serogroup B meningococcal vaccine. 
requiring medical attendance and all serious adverse events (such as admission to hospital) were recorded for the duration of the study.

\section{Statistical analysis}

All immunogenicity analyses are purely descriptive. For each strain, we calculated the proportion of children with protective antibody titres, along with 2-sided 95\% Clopper-Pearson confidence intervals (CIs). Geometric mean titre antibody data (with $95 \%$ CIs) were $\log _{10}$-transformed for analysis.

We analyzed the data on a modified intentionto-treat basis, that is, using data for all enrolled

Table 1: Proportion of children with human serum bactericidal antibody titre of 1:4 or greater at various time points

\begin{tabular}{|c|c|c|c|c|c|c|c|c|}
\hline \multirow[b]{2}{*}{ Participant group* } & \multicolumn{8}{|c|}{ Indicator strain;† $\%$ of children $(95 \% \mathrm{Cl})$} \\
\hline & $44 / 76-S L$ & $5 / 99$ & NZ98/254 & M10713 & UKP1.7-2,4 & GB101 & GB355 & GB364 \\
\hline \multicolumn{9}{|l|}{ 4CMenB $2,4,6,12,40$} \\
\hline After 40-mo booster & $\begin{array}{c}100 \\
(79-100) \\
n=16\end{array}$ & $\begin{array}{c}100 \\
(78-100) \\
n=15\end{array}$ & $\begin{array}{c}88 \\
(62-98) \\
n=16\end{array}$ & $\begin{array}{c}93 \\
(68-100) \\
n=15\end{array}$ & $\begin{array}{c}100 \\
(78-100) \\
n=15\end{array}$ & $\begin{array}{c}100 \\
(78-100) \\
n=15\end{array}$ & $\begin{array}{c}53 \\
(27-79) \\
n=15\end{array}$ & $\begin{array}{c}93 \\
(68-100) \\
n=15\end{array}$ \\
\hline At $60 \mathrm{mo} \ddagger$ & $\begin{array}{c}44 \\
(20-70) \\
n=16\end{array}$ & $\begin{array}{c}88 \\
(62-98) \\
n=16\end{array}$ & $\begin{array}{c}69 \\
(41-89) \\
n=16\end{array}$ & $\begin{array}{c}88 \\
(62-98) \\
n=16\end{array}$ & $\begin{array}{c}81 \\
(54-96) \\
n=16\end{array}$ & $\begin{array}{c}50 \\
(25-75) \\
n=16\end{array}$ & $\begin{array}{c}13 \\
(2-38) \\
n=16\end{array}$ & $\begin{array}{c}81 \\
(54-96) \\
n=16\end{array}$ \\
\hline \multicolumn{9}{|l|}{ rMenB 2,4,6, 12,40 } \\
\hline After 40 -mo booster & $\begin{array}{c}100 \\
(85-100) \\
n=23\end{array}$ & $\begin{array}{c}100 \\
(85-100) \\
n=23\end{array}$ & $\begin{array}{c}17 \\
(5-39) \\
n=23\end{array}$ & $\begin{array}{c}100 \\
(85-100) \\
n=23\end{array}$ & $\begin{array}{c}74 \\
(52-90) \\
n=23\end{array}$ & $\begin{array}{c}100 \\
(85-100) \\
n=23\end{array}$ & $\begin{array}{c}30 \\
(13-53) \\
n=23\end{array}$ & $\begin{array}{c}78 \\
(56-93) \\
n=23\end{array}$ \\
\hline At $60 \mathrm{mo} \neq$ & $\begin{array}{c}46 \\
(26-67) \\
n=24\end{array}$ & $\begin{array}{c}83 \\
(61-95) \\
n=23\end{array}$ & $\begin{array}{c}0 \\
(0-14) \\
n=24\end{array}$ & $\begin{array}{c}77 \\
(55-92) \\
n=22\end{array}$ & $\begin{array}{c}29 \\
(13-51) \\
n=24\end{array}$ & $\begin{array}{c}39 \\
(20-61) \\
n=23\end{array}$ & $\begin{array}{c}0 \\
(0-15) \\
n=23\end{array}$ & $\begin{array}{c}59 \\
(36-79) \\
n=22\end{array}$ \\
\hline \multicolumn{9}{|l|}{ 4CMenB $12,40,42$} \\
\hline After immunization at $42 \mathrm{mo}$ & $\begin{array}{c}100 \\
(48-100) \\
n=5\end{array}$ & $\begin{array}{c}100 \\
(48-100) \\
n=5\end{array}$ & $\begin{array}{c}100 \\
(48-100) \\
n=5\end{array}$ & $\begin{array}{c}100 \\
(48-100) \\
n=5\end{array}$ & $\begin{array}{c}100 \\
(48-100) \\
n=5\end{array}$ & $\begin{array}{c}100 \\
(48-100) \\
n=5\end{array}$ & $\begin{array}{c}100 \\
(48-100) \\
n=5\end{array}$ & $\begin{array}{c}100 \\
(48-100) \\
n=5\end{array}$ \\
\hline At $60 \mathrm{mo}$ & $\begin{array}{c}80 \\
(28-99) \\
n=5\end{array}$ & $\begin{array}{c}100 \\
(48-100) \\
n=5\end{array}$ & $\begin{array}{c}80 \\
(28-99) \\
n=5\end{array}$ & $\begin{array}{c}100 \\
(48-100) \\
n=5\end{array}$ & $\begin{array}{c}100 \\
(48-100) \\
n=5\end{array}$ & $\begin{array}{c}100 \\
(48-100) \\
n=5\end{array}$ & $\begin{array}{c}60 \\
(15-95) \\
n=5\end{array}$ & $\begin{array}{c}80 \\
(28-99) \\
n=5\end{array}$ \\
\hline \multicolumn{9}{|l|}{ rMenB $12,40,42$} \\
\hline After immunization at $42 \mathrm{mo}$ & $\begin{array}{c}100 \\
(75-100) \\
n=13\end{array}$ & $\begin{array}{c}100 \\
(75-100) \\
n=13\end{array}$ & $\begin{array}{c}15 \\
(2-45) \\
n=13\end{array}$ & $\begin{array}{c}83 \\
(52-98) \\
n=12\end{array}$ & $\begin{array}{c}42 \\
(15-72) \\
n=12\end{array}$ & $\begin{array}{c}100 \\
(74-100) \\
n=12\end{array}$ & $\begin{array}{c}8 \\
(0-38) \\
n=12\end{array}$ & $\begin{array}{c}100 \\
(74-100) \\
n=12\end{array}$ \\
\hline At $60 \mathrm{mo}$ & $\begin{array}{c}85 \\
(55-98) \\
n=13\end{array}$ & $\begin{array}{c}100 \\
(75-100) \\
n=13\end{array}$ & $\begin{array}{c}0 \\
(0-25) \\
n=13\end{array}$ & $\begin{array}{c}92 \\
(62-100) \\
n=12\end{array}$ & $\begin{array}{c}9 \\
(0-41) \\
n=11\end{array}$ & $\begin{array}{c}42 \\
(15-72) \\
n=12\end{array}$ & $\begin{array}{c}9 \\
(0-41) \\
n=11\end{array}$ & $\begin{array}{c}83 \\
(52-98) \\
n=12\end{array}$ \\
\hline \multicolumn{9}{|l|}{ 4CMenB 40,42 } \\
\hline After immunization at 40 and $42 \mathrm{mo}$ & $\begin{array}{c}100 \\
(87-100) \\
n=27\end{array}$ & $\begin{array}{c}100 \\
(87-100) \\
n=27\end{array}$ & $\begin{array}{c}93 \\
(76-99) \\
n=27\end{array}$ & $\begin{array}{c}89 \\
(71-98) \\
n=27\end{array}$ & $\begin{array}{c}92 \\
(75-99) \\
n=26\end{array}$ & $\begin{array}{c}100 \\
(87-100) \\
n=26\end{array}$ & $\begin{array}{c}69 \\
(48-86) \\
n=26\end{array}$ & $\begin{array}{c}100 \\
(87-100) \\
n=26\end{array}$ \\
\hline At $60 \mathrm{mo} \neq$ & $\begin{array}{c}71 \\
(51-87) \\
n=28\end{array}$ & $\begin{array}{c}100 \\
(88-100) \\
n=28\end{array}$ & $\begin{array}{c}31 \\
(15-51) \\
n=29\end{array}$ & $\begin{array}{c}81 \\
(62-94) \\
n=27\end{array}$ & $\begin{array}{c}44 \\
(25-65) \\
n=27\end{array}$ & $\begin{array}{c}74 \\
(54-89) \\
n=27\end{array}$ & $\begin{array}{c}41 \\
(22-61) \\
n=27\end{array}$ & $\begin{array}{c}81 \\
(62-94) \\
n=27\end{array}$ \\
\hline \multicolumn{9}{|l|}{ 4CMenB 60,62 } \\
\hline At $60 \mathrm{mo}$, before immunization & $\begin{array}{c}33 \\
(20-48) \\
n=46\end{array}$ & $\begin{array}{c}2 \\
(0.055-12) \\
n=46\end{array}$ & $\begin{array}{c}2 \\
(0.055-12) \\
n=46\end{array}$ & $\begin{array}{c}83 \\
(69-92) \\
n=46\end{array}$ & $\begin{array}{c}9 \\
(2-21) \\
n=46\end{array}$ & $\begin{array}{c}35 \\
(21-50) \\
n=46\end{array}$ & $\begin{array}{c}2 \\
(0.055-12) \\
n=46\end{array}$ & $\begin{array}{c}24 \% \\
(13-39) \\
n=46\end{array}$ \\
\hline After immunization at 60 and $62 \mathrm{mo}$ & $\begin{array}{c}92 \\
(79-98) \\
n=39\end{array}$ & $\begin{array}{c}100 \\
(91-100) \\
n=39\end{array}$ & $\begin{array}{c}100 \\
(91-100) \\
n=39\end{array}$ & $\begin{array}{c}100 \\
(91-100) \\
n=39\end{array}$ & $\begin{array}{c}100 \\
(91-100) \\
n=39\end{array}$ & $\begin{array}{c}95 \\
(83-99) \\
n=39\end{array}$ & $\begin{array}{c}59 \\
(42-74) \\
n=39\end{array}$ & $\begin{array}{c}97 \\
(87-100) \\
n=39\end{array}$ \\
\hline \multicolumn{9}{|c|}{ 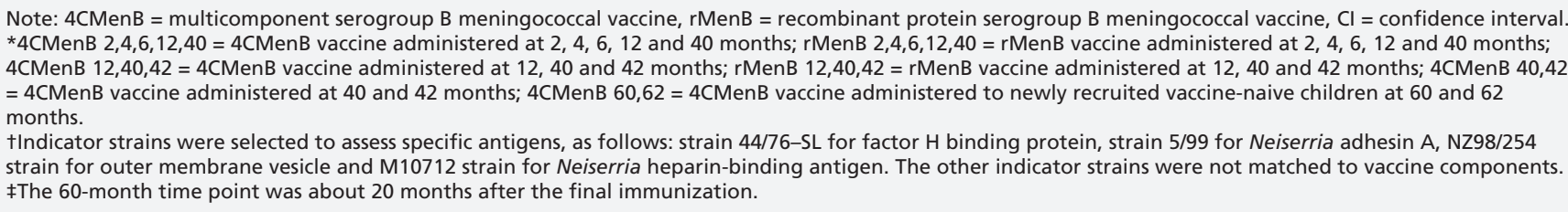 } \\
\hline
\end{tabular}


participants from the original study who provided an evaluable sample at 60 months and all participants from the $4 \mathrm{CMenB} 60,62$ group who received at least 1 vaccination and provided an evaluable sample at either 1 month after the first dose or 1 month after the second dose.

All of the 4 CMenB 60,62 participants received at least 1 dose of the $4 \mathrm{CMenB}$ vaccine and were included in the safety analyses.

\section{Results}

Figure 1 shows the number of children from each group who participated in both the original

Table 2: Geometric mean titres at various time points

\begin{tabular}{|c|c|c|c|c|c|c|c|c|}
\hline \multirow[b]{2}{*}{ Participant group* } & \multicolumn{8}{|c|}{ Indicator strain;† geometric mean titre $(95 \% \mathrm{Cl})$} \\
\hline & 44/76-SL & $5 / 99$ & NZ98/254 & M10713 & UK P1.7-2,4 & GB101 & GB355 & GB364 \\
\hline \multicolumn{9}{|l|}{ 4CMenB $2,4,6,12,40$} \\
\hline After 40-mo booster & $\begin{array}{c}91 \\
(55-151) \\
n=16\end{array}$ & $\begin{array}{c}1786 \\
(860-3708) \\
n=15\end{array}$ & $\begin{array}{c}53 \\
(26-106) \\
n=16\end{array}$ & $\begin{array}{c}40 \\
(24-67) \\
n=15\end{array}$ & $\begin{array}{c}80 \\
(36-181) \\
n=15\end{array}$ & $\begin{array}{c}163 \\
(78-338) \\
n=15\end{array}$ & $\begin{array}{c}5.18 \\
(2.98-9) \\
n=15\end{array}$ & $\begin{array}{c}137 \\
(51-371) \\
n=15\end{array}$ \\
\hline At $60 \mathrm{mo}$ & $\begin{array}{c}4.68 \\
(2.3-9.52) \\
n=16\end{array}$ & $\begin{array}{c}136 \\
(51-365) \\
n=16\end{array}$ & $\begin{array}{c}4.95 \\
(3.54-6.92) \\
n=16\end{array}$ & $\begin{array}{c}10 \\
(5.67-19) \\
n=16\end{array}$ & $\begin{array}{c}8.91 \\
(4.88-16) \\
n=16\end{array}$ & $\begin{array}{c}3.43 \\
(2.04-5.76) \\
n=16\end{array}$ & $\begin{array}{c}1.66 \\
(1.13-2.44) \\
n=16\end{array}$ & $\begin{array}{c}39 \\
(15-99) \\
n=16\end{array}$ \\
\hline \multicolumn{9}{|l|}{ rMenB $2,4,6,12,40$} \\
\hline After 40-mo booster & $\begin{array}{c}107 \\
(70-163) \\
n=23\end{array}$ & $\begin{array}{c}819 \\
(454-1477) \\
n=23\end{array}$ & $\begin{array}{c}1.74 \\
(0.97-3.13) \\
n=23\end{array}$ & $\begin{array}{c}46 \\
(30-69) \\
n=23\end{array}$ & $\begin{array}{c}6.86 \\
(3.56-13) \\
n=23\end{array}$ & $\begin{array}{c}64 \\
(36-116) \\
n=23\end{array}$ & $\begin{array}{c}1.88 \\
(1.2-2.93) \\
n=23\end{array}$ & $\begin{array}{c}41 \\
(18-91) \\
n=23\end{array}$ \\
\hline At $60 \mathrm{mo}$ & $\begin{array}{c}3.13 \\
(1.75-5.59) \\
n=24\end{array}$ & $\begin{array}{c}43 \\
(19-99) \\
n=23\end{array}$ & $\begin{array}{c}1.05 \\
(0.8-1.38) \\
n=24\end{array}$ & $\begin{array}{c}12 \\
(7.22-20) \\
n=22\end{array}$ & $\begin{array}{c}1.76 \\
(1.08-2.87) \\
n=24\end{array}$ & $\begin{array}{c}2.16 \\
(1.4-3.33) \\
n=23\end{array}$ & $\begin{array}{c}1 \\
(0.73-1.38) \\
n=23\end{array}$ & $\begin{array}{c}8.74 \\
(3.94-19) \\
n=22\end{array}$ \\
\hline \multicolumn{9}{|l|}{ 4CMenB $12,40,42$} \\
\hline After immunization at $42 \mathrm{mo}$ & $\begin{array}{c}136 \\
(62-299) \\
n=5\end{array}$ & $\begin{array}{c}3351 \\
(1537-7305) \\
n=5\end{array}$ & $\begin{array}{c}79 \\
(23-274) \\
n=5\end{array}$ & $\begin{array}{c}28 \\
(6.99-112) \\
n=5\end{array}$ & $\begin{array}{c}69 \\
(21-228) \\
n=5\end{array}$ & $\begin{array}{c}113 \\
(40-320) \\
n=5\end{array}$ & $\begin{array}{c}12 \\
(6.02-25) \\
n=5\end{array}$ & $\begin{array}{c}227 \\
(45-1146) \\
n=5\end{array}$ \\
\hline At $60 \mathrm{mo}$ & $\begin{array}{c}13 \\
(3.52-45) \\
n=5\end{array}$ & $\begin{array}{c}210 \\
(36-1227) \\
n=5\end{array}$ & $\begin{array}{c}11 \\
(5.93-20) \\
n=5\end{array}$ & $\begin{array}{c}25 \\
(8.47-74) \\
n=5\end{array}$ & $\begin{array}{c}34 \\
(11-98) \\
n=5\end{array}$ & $\begin{array}{c}11 \\
(4.53-29) \\
n=5\end{array}$ & $\begin{array}{c}4.68 \\
(2.36-9.28) \\
n=5\end{array}$ & $\begin{array}{c}20 \\
(3.78-107) \\
n=5\end{array}$ \\
\hline \multicolumn{9}{|l|}{ rMenB $12,40,42$} \\
\hline After immunization at $42 \mathrm{mo}$ & $\begin{array}{c}127 \\
(78-207) \\
n=13\end{array}$ & $\begin{array}{c}5240 \\
(3232-8497) \\
n=13\end{array}$ & $\begin{array}{c}1.86 \\
(0.86-4.03) \\
n=13\end{array}$ & $\begin{array}{c}21 \\
(8.56-51) \\
n=12\end{array}$ & $\begin{array}{c}3.03 \\
(1.4-6.55) \\
n=12\end{array}$ & $\begin{array}{c}43 \\
(22-85) \\
n=12\end{array}$ & $\begin{array}{c}1.29 \\
(0.81-2.05) \\
n=12\end{array}$ & $\begin{array}{c}616 \\
(217-1750) \\
n=12\end{array}$ \\
\hline At $60 \mathrm{mo}$ & $\begin{array}{c}18 \\
(8.08-39) \\
n=13\end{array}$ & $\begin{array}{c}369 \\
(123-1103) \\
n=13\end{array}$ & $\begin{array}{c}1 \\
(0.69-1.45) \\
n=13\end{array}$ & $\begin{array}{c}12 \\
(5.85-24) \\
n=12\end{array}$ & $\begin{array}{c}1.39 \\
(0.67-2.88) \\
n=11\end{array}$ & $\begin{array}{c}3.01 \\
(1.65-5.47) \\
n=12\end{array}$ & $\begin{array}{c}1.35 \\
(0.85-2.14) \\
n=11\end{array}$ & $\begin{array}{c}39 \\
(13-114) \\
n=12\end{array}$ \\
\hline \multicolumn{9}{|l|}{ 4CMenB 40,42 } \\
\hline $\begin{array}{l}\text { After immunization at } 40 \text { and } \\
42 \mathrm{mo}\end{array}$ & $\begin{array}{c}82 \\
(57-117) \\
n=27\end{array}$ & $\begin{array}{c}888 \\
(645-1222) \\
n=27\end{array}$ & $\begin{array}{c}42 \\
(24-72) \\
n=27\end{array}$ & $\begin{array}{c}38 \\
(23-62) \\
n=27\end{array}$ & $\begin{array}{c}29 \\
(19-44) \\
n=26\end{array}$ & $\begin{array}{c}192 \\
(98-376) \\
n=26\end{array}$ & $\begin{array}{c}7.39 \\
(3.76-14) \\
n=26\end{array}$ & $\begin{array}{c}103 \\
(68-157) \\
n=26\end{array}$ \\
\hline At $60 \mathrm{mo}$ & $\begin{array}{c}12 \\
(6.27-23) \\
n=28\end{array}$ & $\begin{array}{c}44 \\
(29-67) \\
n=28\end{array}$ & $\begin{array}{c}2.42 \\
(1.59-3.66) \\
n=29\end{array}$ & $\begin{array}{c}8.52 \\
(5.09-14) \\
n=27\end{array}$ & $\begin{array}{c}2.87 \\
(1.96-4.18) \\
n=27\end{array}$ & $\begin{array}{c}11 \\
(5.4-22) \\
n=27\end{array}$ & $\begin{array}{c}2.93 \\
(1.74-4.93) \\
n=27\end{array}$ & $\begin{array}{c}15 \\
(9.06-25) \\
n=27\end{array}$ \\
\hline \multicolumn{9}{|l|}{ 4CMenB 60,62 } \\
\hline $\begin{array}{l}\text { At } 60 \text { mo, before } \\
\text { immunization }\end{array}$ & $\begin{array}{c}2.98 \\
(1.86-4.78) \\
n=46\end{array}$ & $\begin{array}{c}1.14 \\
(0.88-1.47) \\
n=46\end{array}$ & $\begin{array}{c}1.04 \\
(0.96-1.14) \\
n=46\end{array}$ & $\begin{array}{c}18 \\
(12-28) \\
n=46\end{array}$ & $\begin{array}{c}1.34 \\
(1.07-1.68) \\
n=46\end{array}$ & $\begin{array}{c}2.27 \\
(1.62-3.18) \\
n=46\end{array}$ & $\begin{array}{c}1.15 \\
(0.96-1.37) \\
n=46\end{array}$ & $\begin{array}{c}2.24 \\
(1.54-3.26) \\
n=46\end{array}$ \\
\hline $\begin{array}{l}\text { After immunization at } 60 \text { and } \\
62 \mathrm{mo}\end{array}$ & $\begin{array}{c}34 \\
(23-50) \\
n=39\end{array}$ & $\begin{array}{c}875 \\
(546-1404) \\
n=39\end{array}$ & $\begin{array}{c}29 \\
(21-41) \\
n=39\end{array}$ & $\begin{array}{c}42 \\
(32-54) \\
n=39\end{array}$ & $\begin{array}{c}37 \\
(26-51) \\
n=39\end{array}$ & $\begin{array}{c}14 \\
(9.92-21) \\
n=39\end{array}$ & $\begin{array}{c}3.99 \\
(2.71-5.87) \\
n=39\end{array}$ & $\begin{array}{c}97 \\
(63-151) \\
n=39\end{array}$ \\
\hline 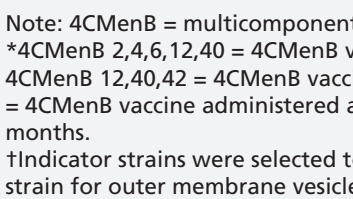 & $\begin{array}{l}\text { roup B men } \\
\text { administer } \\
\text { ninistered a } \\
\text { nd } 42 \text { mont } \\
\text { s specific an } \\
\text { M10712 stra }\end{array}$ & $\begin{array}{l}\text { coccal vaccir } \\
2,4,6,12 \text { a } \\
40 \text { and } 42 n \\
\text { MenB } 60,62 \\
\text { S, as follow } \\
\text { Neiserria } \mathrm{h}\end{array}$ & $\begin{array}{l}\text { lenB }=\text { recor } \\
\text { months; } r N \\
\text { s; rMenB } 12 \\
\text { MenB vaccir } \\
\text { in 44/76-SL } \\
\text { n-binding ar }\end{array}$ & $\begin{array}{l}\text { nant prote } \\
\mathrm{B} 2,4,6,12, \\
42=\mathrm{rMer} \\
\text { administer } \\
\text { factor } \mathrm{H} \mathrm{k} \\
\text { en. The o }\end{array}$ & $\begin{array}{l}\text { rogroup B r } \\
\text { rMenB vacc } \\
\text { ccine admin } \\
\text { newly recr } \\
\text { g protein, }\end{array}$ & $\begin{array}{l}\text { ngococcal } \\
\text { idminister } \\
\text { ed at } 12,4 \\
\text { i vaccine-n } \\
5 / 99 \text { for } \\
\text { were not }\end{array}$ & $\begin{array}{l}\text { ne, } \mathrm{Cl}=\text { con } \\
2,4,6,12 \text { ar } \\
\text { d } 42 \text { months } \\
\text { children at } 6 \\
\text { rria adhesin } \\
\text { eed to vaccir }\end{array}$ & $\begin{array}{l}\text { ence interval. } \\
40 \text { months; } \\
\text { CMenB } 40,42 \\
\text { and } 62 \\
\text { NZ98/254 } \\
\text { components. }\end{array}$ \\
\hline
\end{tabular}


study and the 40- and 60-month stages of the follow-on study. Between enrolment in the follow-on study (at $40 \mathrm{mo}$ ) and blood sampling at 60 months, 9 children were lost to follow up, 5 withdrew consent (mainly because further blood tests were declined), and 3 moved out of the area; in addition, it was determined that 1 child had been inappropriately enrolled at 40 months. Overall (among the 163 participants enrolled in the 40-month follow-on study or newly recruited at 60 months of age), 79 (48\%) were boys and $84(52 \%)$ were girls. Self-defined ethnicity was

Table 3: Children experiencing reactions in the 7 days after each dose of vaccine, among vaccine-naive participants receiving the first dose at 60 months of age (for dose 1, $n=50$; for dose $2, n=46$ )

\begin{tabular}{|c|c|c|c|c|c|c|c|c|}
\hline \multirow[b]{3}{*}{ Reaction } & \multicolumn{8}{|c|}{ Severity of reaction; ${ }^{*}$ dose number; no. (\%) of children) } \\
\hline & \multicolumn{2}{|c|}{ Mild } & \multicolumn{2}{|c|}{ Moderate } & \multicolumn{2}{|c|}{ Severe } & \multicolumn{2}{|c|}{ Overallt } \\
\hline & Dose 1 & Dose 2 & Dose 1 & Dose 2 & Dose 1 & Dose 2 & Dose 1 & Dose 2 \\
\hline \multicolumn{7}{|l|}{ Solicited local } & 49 (98) & $45(98)$ \\
\hline Pain & $17(34)$ & $10(22)$ & $17(34)$ & $27(59)$ & $6(12)$ & $2(4)$ & & \\
\hline Erythema & $37(74)$ & $36(78)$ & $6(12)$ & $3(7)$ & $4(8)$ & $1(2)$ & & \\
\hline Swelling & $14(28)$ & $18(39)$ & $3(6)$ & $1(2)$ & $2(4)$ & $0(0)$ & & \\
\hline Induration & $19(38)$ & $19(41)$ & $4(8)$ & $0(0)$ & $0(0)$ & $0(0)$ & & \\
\hline \multicolumn{7}{|l|}{ Solicited systemic } & $35(70)$ & $35(76)$ \\
\hline Change in appetite & $14(28)$ & $13(28)$ & $3(6)$ & $2(4)$ & $0(0)$ & $1(2)$ & & \\
\hline Sleepiness & $15(30)$ & $9(20)$ & $3(6)$ & $5(11)$ & $0(0)$ & $1(2)$ & & \\
\hline Vomiting & $2(4)$ & $1(2)$ & $3(6)$ & $2(4)$ & $0(0)$ & $1(2)$ & & \\
\hline Diarrhea & $4(8)$ & $4(9)$ & $0(0)$ & $0(0)$ & $0(0)$ & $0(0)$ & & \\
\hline Irritability & $15(30)$ & $14(30)$ & $9(18)$ & $5(11)$ & $0(0)$ & $1(2)$ & & \\
\hline Headache & $3(6)$ & $2(4)$ & $2(4)$ & $1(2)$ & $0(0)$ & $0(0)$ & & \\
\hline Arthralgia & $5(10)$ & $5(11)$ & $4(8)$ & $7(15)$ & $1(2)$ & $0(0)$ & & \\
\hline Rash & $2(4)$ & $3(7)$ & $0(0)$ & $0(0)$ & $0(0)$ & $0(0)$ & & \\
\hline Fever (temperature $>38^{\circ} \mathrm{C}$ ) & $2(4)$ & $1(2)$ & $3(6)$ & $1(2)$ & $0(0)$ & $0(0)$ & & \\
\hline \multicolumn{7}{|l|}{ Other adverse eventsł } & $4(8)$ & $5(11)$ \\
\hline Pain $>7 \mathrm{~d} \S$ & $0(0)$ & $1(2)$ & $0(0)$ & $0(0)$ & $0(0)$ & $0(0)$ & & \\
\hline Erythema $>7 \mathrm{~d} \S$ & $1(2)$ & $1(2)$ & $0(0)$ & $0(0)$ & $0(0)$ & $0(0)$ & & \\
\hline Swelling $>7 \mathrm{~d} \S$ & $1(2)$ & $2(4)$ & $0(0)$ & $0(0)$ & $0(0)$ & $0(0)$ & & \\
\hline Induration $>7 \mathrm{~d} \S$ & $1(2)$ & $2(4)$ & $0(0)$ & $0(0)$ & $0(0)$ & $0(0)$ & & \\
\hline Abdominal pain & $1(2)$ & $1(2)$ & $0(0)$ & $0(0)$ & $0(0)$ & $0(0)$ & & \\
\hline Injection-site reaction & $1(2)$ & $0(0)$ & $0(0)$ & $0(0)$ & $0(0)$ & $0(0)$ & & \\
\hline Tonsillitis & $1(2)$ & $0(0)$ & $0(0)$ & $0(0)$ & $0(0)$ & $0(0)$ & & \\
\hline Nausea & $0(0)$ & $1(2)$ & $0(0)$ & $0(0)$ & $0(0)$ & $0(0)$ & & \\
\hline Cough & $0(0)$ & $1(2)$ & $0(0)$ & $0(0)$ & $0(0)$ & $0(0)$ & & \\
\hline \multicolumn{9}{|l|}{ Serious adverse event } \\
\hline \multicolumn{4}{|l|}{$\begin{array}{l}\text { Admission to hospital, } \\
\text { death, permanent disability }\end{array}$} & accine (se & ext for $d e$ & & \multicolumn{2}{|c|}{2 (4)ף } \\
\hline \multicolumn{9}{|c|}{$\begin{array}{l}\text { *Mild = no limitation to normal activity (for pain, sleepiness, arthralgia, headache, irritability), } 1-25 \mathrm{~mm} \text { (for erythema, swelling, induration), complete meals } \\
\text { twice a day (for change in appetite), } 1 \text { or } 2 \text { vomits } / \text { day (for vomiting), } 2 \text { or } 3 \text { loose stools } / \text { day (for diarrhea), temperature } 38-38.9^{\circ} \mathrm{C}(\text { for fever); moderate }=5 \text { some } \\
\text { limitation to normal activity, } 25-50 \mathrm{~mm} \text {, missed } 1 \text { or } 2 \text { meals a day, more than } 2 \text { vomits } / \text { day, } 4 \text { or } 5 \text { loose stools/day, temperature } 39-39.9^{\circ} \mathrm{C} ; \text { severe }=\text { unable to } \\
\text { perform normal activity, }>50 \mathrm{~mm} \text {, no meals, vomits requiring intravenous hydration, } 6 \text { or more loose stools or required intravenous hydration, temperature } \\
\geq 40^{\circ} \mathrm{C} \text {. } \\
\text { †Some participants had more than one reaction. } \\
\text { ¥At least possibly related to vaccination. } \\
\text { \$Pain, erythema, induration and swelling lasting more than } 7 \text { days were classified as separate adverse events. } \\
\text { IBoth of these events occurred } 2 \text { months after the second dose and were judged to be unrelated to the vaccine. }\end{array}$} \\
\hline
\end{tabular}


$152(93 \%)$ white, 3 (2\%) black, $1(1 \%)$ Asian and $7(4 \%)$ "other." Within the newly recruited control group, the mean age at enrolment was 61.3 (range 59-63) months.

\section{Persistence of immunity}

The percentage of children in each strain analysis group with hSBA of 1:4 or greater at 60 months of age is shown in Table 1 and Appendix 1 (available at www.cmaj.ca/lookup/suppl /doi:10.1503/cmaj.141200/-/DC1). The geometric mean titres are shown in Table 2 and Appendix 2 (available at www.cmaj.ca/lookup/suppl /doi:10.1503/cmaj.141200/-/DC1). Waning of antibody titres was observed for those immunized as infants, but the extent of waning varied by strain. For half of the 8 strains tested, hSBA geometric mean titres and the proportion of participants with antibody titres of 1:4 or greater were similar among those who had received $4 \mathrm{CMenB}$ at 2, 4, 6, 12 and 40 months and those who had received no vaccination (Table 1 ; hSBA $\geq 1: 4$ at 60 months for strain 44/76-SL, $44 \%$ v. $33 \%$; for strain M10713, 88\% v. 83\%; for strain GB101, $50 \%$ v. $35 \%$; and for strain GB355, $13 \%$ v. $2 \%$ ). When compared with vaccine-naive controls, a higher percentage of participants who had previously received the $4 \mathrm{CMenB}$ vaccine had hSBA of 1:4 or greater for strains $5 / 99(88 \%-100 \% \mathrm{v}$. $2 \%$ ), NZ98/254 (31\%-80\% v. 2\%), UKP1.7-2.4 (44\%-100\% v. 9\%), GB355 (41\%-60\% [except the 4 CMenB 2,4,6,12,40 group, which was $13 \%$ ] v. $2 \%)$ and GB364 (80\%-81\% v. $24 \%$, although 95\% CIs overlapped between the 4CMenB $12,40,42$ and vaccine-naive groups) (Table 1).

The results for the rMenB group followed a similar pattern, except that no hSBA response against strain NZ98/254 was observed at 60 months. This result was to be expected, given the lack of an outer membrane vesicle component in the rMenB vaccine.

\section{Response to immunization at 60 and 62 months}

The hSBA response to administration of the $4 \mathrm{CMenB}$ vaccine in the formerly vaccine-naive participants (group $4 \mathrm{CMenB} 60,62$ ) is shown in Tables 1 and 2 and in Appendices 1 and 2 . Postimmunization hSBA titres of 1:4 or greater were observed for all strains for $92 \%-100 \%$ of participants, with the notable exception of strain GB355, for which 59\% of participants developed antibodies above the correlate of protection.

\section{Reactogenicity at $\mathbf{6 0}$ months}

Overall, 49 (98\%) of the 50 participants who received the $4 C M e n B$ vaccine at 60 and 62 months experienced at least 1 solicited local adverse event, with erythema being the most commonly experienced local reaction (Table 3 ). Severe pain was reported by $12 \%$ (6/50) of participants after dose 1 and 4\% (2/46) after dose 2. By day 3, the pain had lessened among all those who experienced severe pain, but $10 \%(5 / 50)$ reported some level of pain for up to 7 days, and $2 \%(1 / 46)$ had pain persisting for more than 7 days after dose 2 . Forty-two (84\%) of the 50 participants had a solicited systemic adverse event, irritability being the most frequent. Fever rates were 10\% (5/50) after dose 1 and 4\% (2/46) after dose 2 . There was 1 case of fever requiring medical attendance.

Eight $(16 \%)$ of the 50 participants experienced a nonserious, unsolicited adverse event that may have been related to the vaccine (Table 3 ). There were 2 serious adverse events requiring admission to hospital; however, neither was judged to be related to the vaccine. In one case, the participant had hematuria secondary to a urinary tract infection 2 months after the last vaccine dose. In the other case, the child had herpetic stomatitis requiring admission to hospital, also at 2 months after the last vaccine dose.

\section{Interpretation}

The results presented here provide important new information about how the persistence, at 5 years of age, of bactericidal activity induced by administration of $4 \mathrm{CMenB}$ vaccine differs between test strains and with different vaccination schedules. In addition, we found that $4 \mathrm{CMenB}$ vaccine was immunogenic when given as a catch-up course to children at 5 years of age; however, the persistence of this immunity into later childhood is unknown. Before immunization, only a small proportion of the vaccinenaive controls had protective antibody titres. A notable exception to this observation was indicator strain M10713, for which $83 \%$ of vaccinenaive participants had bactericidal activity. This finding may suggest exposure to a cross-reactive antigen or may indicate that this strain is highly prevalent but not particularly virulent or that it is more sensitive to killing in the hSBA assay when tested with sera in this age group.

The data summarized here, from both the current study and the original trial, ${ }^{7}$ also suggest that a reduction in the number of doses given to infants may be cost-effective without compromising immunogenicity and disease prevention, a particularly pertinent issue for the $4 \mathrm{CMenB}$ vaccine, given discussions about its cost-effectiveness. ${ }^{12}$ With the exception of strains NZ98/254 and GB101, there was minimal increase in geometric mean titres (Appendix 2) or the proportion of children with 
protective antibody titres (Appendix 1) between samples taken at 5 and 7 months (1 mo after the second and third doses, respectively). ${ }^{7}$ A late 2-dose schedule for infants (with vaccine administered at 6 and $8 \mathrm{mo}$ ) has previously been shown to be immunogenic, ${ }^{13}$ and this schedule is now being evaluated in larger trials in early infancy (Clinical Trials.gov identifier NCT01339923). However, it is important that consideration be given to the practicalities of delivering such a schedule, as there is currently no routine immunization visit at 8 months of age in either the UK or Canada.

The value of an additional preschool booster dose of the $4 \mathrm{CMenB}$ vaccine, as used in the original follow-on study, ${ }^{9}$ is also not yet clear. It is important to maintain protection against serogroup B meningococcal disease throughout the first few years of life, when disease incidence is high, ${ }^{2}$ but the additional increase in proportion of children with antibody titres of 1:4 or greater after a 40-month booster dose appears temporary and may not outweigh the cost of implementation at this age. When the 2 groups with doses administered at 40 and 42 months (4CMenB 12,40,42 and 4CMenB 40,42) are compared, there seems to be no advantage for the group who received an additional priming dose at 12 months, in terms of either geometric mean titres or percentage with hSBA titres of 1:4 or greater at 43 months ( 1 mo after last vaccine). ${ }^{9}$ However, children receiving the vaccine at 40 and 42 months or at 60 and 62 months will not have been protected as young infants, when the incidence of serogroup B meningococcal disease is highest. ${ }^{2}$ Given that $56 \%$ of UK cases occur under the age of 5 years, the total disease burden may not be significantly reduced with later immunization. ${ }^{4}$

Given these findings, an infant schedule followed by boosting in adolescence may be preferable. One study of adolescents has been published, in which 11- to 17-year-olds were followed for 18 to 24 months after receiving 1 , 2 or 3 doses of the $4 \mathrm{CMenB}$ vaccine. ${ }^{14}$ The geometric mean titre response, both initially and 20 months after the last dose, was substantially higher in these adolescents than in the children in the current study for strains 44/76-SL (20-mo geometric mean titre 13 v. 44 for infant and adolescent groups who received 3 doses) and NZ98/254 (20-mo geometric mean titre 11 v. 30 for 3-dose groups), but this difference was not seen for strain $5 / 99$, and other strains were not tested. Both initial response and persistence may be better among adolescents, but further studies directly comparing the 2 age groups are required to confirm this difference, and the issue of directly protecting high-risk infants remains.

\section{Limitations}

This study was limited in terms of sample size, particularly for the $4 \mathrm{CMenB} 12,40,42$ group, of whom only 5 of the original 24 children were evaluated at 60 months. The lack of an unimmunized control group also limits the interpretation of reactogenicity at 60 and 62 months. Nevertheless, these data give an early indication that protection against serogroup B meningococcal disease after infant immunization is unlikely to persist into adolescence, when the second peak of incidence occurs. ${ }^{2}$ The steep decline in disease incidence after the first year of life $^{2}$ means that schedules involving vaccination at 40 and 42 months and at 60 and 62 months may not have a substantial effect on the number of cases and are therefore less likely to be used if the incidence of disease remains at its current low levels. However, these data will prove useful in planning for a future epidemic or for countries planning catch-up campaigns.

\section{Conclusion}

There remains a need for more information about the effects of the $4 \mathrm{CMenB}$ vaccine on nasopharyngeal carriage, the likely breadth of strain coverage and long-term persistence. Data from studies using the Meningococcal Antigen Typing System, which aims to predict overall strain coverage, suggest that up to $88 \%$ of UK strains ${ }^{15}$ and $66 \%$ of Canadian strains ${ }^{16}$ will be covered by the $4 \mathrm{CMenB}$ vaccine; however, the key test will be how the vaccine performs when administered routinely to whole populations. The Saguenay-Lac-Saint-Jean region of Quebec, which has relatively high rates of serogroup B meningococcal disease, recently ran a vaccine campaign using the $4 \mathrm{CMenB}$ vaccine ${ }^{17}$ however, the Public Health Agency of Canada currently recommends vaccination only for those in defined high-risk groups. ${ }^{5}$ Introduction of the $4 \mathrm{CMenB}$ vaccine into the UK's routine immunization schedule provides an ideal opportunity to assess the effect of this vaccine in a real-world setting and will guide the implementation of $4 \mathrm{CMenB}$ vaccination in Canada and worldwide.

\section{References}

1. Andrews SM, Pollard AJ. A vaccine against serogroup B Neisseria meningitidis: dealing with uncertainty. Lancet Infect Dis 2014; 14:426-34.

2. Ladhani SN, Flood JS, Ramsay ME, et al. Invasive meningococcal disease in England and Wales: implications for the introduction of new vaccines. Vaccine 2012;30:3710-6.

3. Viner RM, Booy R, Johnson H, et al. Outcomes of invasive meningococcal serogroup B disease in children and adolescents (MOSAIC): a case-control study. Lancet Neurol 2012;11:774-83.

4. Invasive meningococcal disease (laboratory reports in England): 2013/2014 annual data by epidemiological year. Health Prot Rep 2015;9(3):1-3. Available: www.gov.uk/government/uploads /system/uploads/attachment_data/file/397913/hpr0315_imd.pdf (accessed 2015 Feb. 21). 
5. Pan-Canadian Public Health Network. The recommended use of the multicomponent meningococcal B (4CMenB) vaccine in Canada. Ottawa: Public Health Agency of Canada; 2014. Available: www.phac-aspc.gc.ca/naci-ccni/mening-4cmenb-exec-resum-eng .php (accessed 2014 Nov. 22).

6. Minutes of the meeting on Tuesday 11 and Wednesday 12 February 2014. Joint Committee on Vaccination and Immunisation; 2014 Available: www.gov.uk/government/groups/joint-committee-on -vaccination-and-immunisation\#minutes (accessed 2014 Nov. 22).

7. Findlow J, Borrow R, Snape MD, et al. Multicenter, open-label, randomized phase II controlled trial of an investigational recombinant meningococcal serogroup B vaccine with and without outer membrane vesicles, administered in infancy. Clin Infect Dis 2010;51:1127-37.

8. Toneatto D, Ismaili S, Ypma E, et al. The first use of an investigational multicomponent meningococcal serogroup $\mathrm{B}$ vaccine (4CMenB) in humans. Hum Vaccin 2011;7:646-53.

9. Snape MD, Saroey P, John TM, et al. Persistence of bactericidal antibodies following early infant vaccination with a serogroup B meningococcal vaccine and immunogenicity of a preschool booster dose. CMAJ 2013;185:E715-24.

10. Martin NG, Snape MD. A multicomponent serogroup B meningococcal vaccine is licensed for use in Europe: What do we know, and what are we yet to learn? Expert Rev Vaccines 2013 12:837-58.

11. Borrow R, Carlone GM, Rosenstein N, et al. Neisseria meningitidis group $\mathrm{B}$ correlates of protection and assay standardization - international meeting report Emory University, Atlanta, Georgia, United States, 16-17 March 2005. Vaccine 2006;24: 5093-107.

12. JCVI position statement on the use of Bexsero® meningococcal $B$ vaccine in the UK. London (UK): Department of Health and Public Health England; 2014. Available: www.gov.uk/government /publications/meningococcal-b-vaccine-jcvi-position-statement (accessed 2014 Nov. 22)

13. Snape MD, Dawson T, Oster P, et al. Immunogenicity of two investigational serogroup B meningococcal vaccines in the first year of life: a randomized comparative trial. Pediatr Infect Dis $J$ 2010;29:e71-9.

14. Santolaya ME, O'Ryan M, Valenzuela MT, et al. Persistence of antibodies in adolescents 18-24 months after immunization with one, two, or three doses of $4 \mathrm{CMenB}$ meningococcal serogroup B vaccine. Hum Vaccin Immunother 2013;9:2304-10

15. Frosi G, Biolchi A, Lo Sapio M, et al. Bactericidal antibody against a representative epidemiological meningococcal serogroup B panel confirms that MATS underestimates 4CMenB vaccine strain coverage. Vaccine 2013;31:4968-74.

16. Bettinger JA, Scheifele DW, Halperin SA, et al. Diversity of Canadian meningococcal serogroup $\mathrm{B}$ isolates and estimated coverage by an investigational meningococcal serogroup $\mathrm{B}$ vaccine (4CMenB). Vaccine 2013;32:124-30.

17. Targeted meningococcal serogroup $B$ vaccination campaign in the Saguenay-Lac-Saint-Jean. Quebec City: Santé et Services sociaux Québec; 2014. Available: www.msss.gouv.qc.ca/sujets/santepub /vaccination/index.php?meningococcal-b-vaccination-campaign (accessed 2015 Feb. 21).

Competing interests: Matthew Snape acts as an investigator for clinical studies for both noncommercial funding bodies and commercial sponsors (Novartis Vaccines, GlaxoSmithKline, Sanofi-Pasteur, Sanofi-PasteurMSD, Pfizer Vaccines and Johnson \& Johnson) conducted on behalf of the University of Oxford. He also undertakes consultancy and advisory work for Novartis Vaccines and Diagnostics, Pfizer and Sanofi-Pasteur. Speaking honoraria and travel and accommodation reimbursements from these organizations are paid to the University of Oxford Department of Paediatrics. Matthew Snape does not receive any personal financial support from vaccine manufacturers. Peter Dull, Daniela Toneatto and Diego D'Agostino are employees of Novartis Vaccines. Daniela Toneatto has stock options from Novartis Vaccines. Hannah Robinson has received reimbursement from Okiros for travel and accommodation expenses for study meetings unrelated to the current work. Andrew Pollard, on behalf of University of Oxford, has previously acted as an investigator on trials of meningococcal vaccines funded by Novartis Vaccines and Pfizer. He has no pecuniary interests in vaccine manufacturers and receives no personal payments from industry. Andrew Pollard is chair of the UK Department of Health's Joint Committee on Vaccination and Immunisation. The views expressed in this manuscript do not necessarily reflect the views of the Department of Health. No other competing interests were declared.

Affiliations: Oxford Vaccine Group, Department of Paediatrics (McQuaid, Snape, John, Kelly, Robinson, Pollard), and Nuffield Department of Primary Care Health Sciences (Yu), University of Oxford, Oxford, UK; Oxford Biomedical Research Centre (McQuaid, Snape, John, Kelly, Robinson, Pollard), National Institute for Health Research, Oxford, UK; Novartis Vaccines and Diagnostics S.r.1. (Toneatto), Siena, Italy; Novartis Pharma BV (D’Agostino), Amsterdam, Netherlands; Novartis Vaccines and Diagnostics Inc. (Dull), Cambridge, Mass.

Contributors: Matthew Snape, Daniela Toneatto, Peter Dull and Andrew Pollard contributed to the study design. Matthew Snape, Tessa John, Sarah Kelly, Hannah Robinson and Andrew Pollard contributed to acquisition of the data. Fiona McQuaid, Matthew Snape, Ly-Mee Yu, Diego D'Agostino and Andrew Pollard contributed to analysis of the data. Fiona McQuaid drafted the manuscript. All authors contributed to revising the article, approved the final version and agree to act as guarantors of the work.

Funding: This study was funded by Novartis Vaccines. The Oxford Biomedical Research Centre of the National Institute for Health Research provides salary support for Matthew Snape and Tessa John. Andrew Pollard is a Jenner Investigator and James Martin Senior Fellow.

Data sharing: Details regarding the sharing of patient-level data are available on the website www.novartis.com/innovation /responsibly-tackling-the-challenging-issues/clinical-trial -information-disclosure.shtml. All results of this study were verified independently by a statistician from the University of Oxford. 